Computing and Informatics, Vol. 38, 2019, 1067 1090 doi: 10.31577/cai_2019_5 1067

\title{
NEW APPROACH TO EDGE DETECTION ON DIFFERENT LEVEL OF WAVELET DECOMPOSITION
}

\author{
Vladimir Maksimović, Branimir JaKŠıć, Mile Petrović \\ Petar SPAlević, Stefan PANić \\ Faculty of Technical Science \\ University of Priština \\ Kneza Miloša 7 \\ 38220 Kosovska Mitrovica, Serbia \\ e-mail: \{vladimir.maksimovic, branimir.jaksic, mile.petrovic, \\ petar.spalevic\}@pr.ac.rs, stefanpnc@tpu.ru
}

\begin{abstract}
This paper proposes a new approach to edge detection on the images over which the wavelet decomposition was done to the third level and consisting of different levels of detail (small, medium and high level of detail). Images from the BSD (Berkeley Segmentation Dataset) database with the corresponding ground truth were used. Daubechies wavelet was used from second to tenth order. Gradient and Laplacian operators were used for edge detection. The proposed approach is applied in systems where information is processed in real time, where fast image processing is required and in systems where high compression ratio is used. That is, it can find practical application in many systems, especially in television systems where the level of details in the image changes. The new approach consists in the fact that when wavelet transform is applied, an edge detection is performed over the level 1 image to create a filter. The filter will record only those pixels that can be potential edges. The image is passed through a median filter that filters only the recorded pixels and 8 neighbors of pixel. After that, the edge detection with one of the operators is applied onto the filtered image. F measure, FoM (Figure of Merit) and PR (Performance Ratio) were used as an objective measure. Based on the obtained results, the application of the proposed approach achieves significant improvements and these improvements are very good depending on the number of details in the image and the compression ratio. These results and improvements can be used to improve the quality of edge detection in many systems where compressed images are processed, that is, where work with images with a high compression ratio is required.
\end{abstract}


Keywords: Edge detection, wavelet decomposition, compression, F measure, figure of merit, performance ratio

\section{INTRODUCTION}

In recent years, multimedia systems have recorded tremendous growth and progress, which means that image resolutions got higher increasing the complexity of the system and the complexity of the image. It is necessary to achieve as much compression as possible so these images can be streamed, stored and processed more easily. Today's trends require more technology to be involved, so in television systems we have a virtual reality (VR), augmented reality (AR) and a combination of them. Since it is necessary to have lower bitrate and achieve a high compression ratio and to process an image in VR or AR environment, it is necessary to perform certain operations over images such as segmentation, edge detection, etc. [1].

Edge detection is one of the fundamental processes in image processing. This also means the segmentation of the image where the segmentation of the desired object is performed by detecting the edge. Edge detection is based on the fact that there are sudden changes in the gray intensity between the objects. Edge detection significantly reduces the image analysis process by using less data and at the same time storing all the necessary information. Many edge detection techniques have been tried, but gradient and Laplacian methods have proved to be the best [2, 3]. Gradient methods for edge detection find the maximum and minimum gradient of the image intensity, all in the first derivative of the image. Laplacian methods are based on finding zero crossing in the second derivative of the image [4]. The gradient of the image can be calculated as [4, 5]:

$$
\nabla f(x, y)=\frac{\partial f}{\partial x} i+\frac{\partial f}{\partial y} j
$$

where $f(x, y)$ represents the image at the location $(x, y)$ where $x$ and $y$ are the coordinates of the row and column. The gradient $\nabla f(x, y)$ contains the information about gray change. The gradient of $\nabla f(x, y)$ can be calculated [4, 5]:

$$
e(x, y)=\sqrt{f_{x}^{2}+f_{y}^{2}}
$$

where $e(x, y)$ can be used as an edge detector and can also be defined as the sum of the absolute values of the partial derivative $f_{x}$ and $f_{y}[\underline{4},[5]$ :

$$
e(x, y)=\left|f_{x}(x, y)\right|+\left|f_{y}(x, y)\right|
$$

Based on these theoretical principles, the gradient and Laplacian methods are proposed. The gradient methods include Sobel, Prewitt, Robert, while the Laplacian include LoG (Laplacian of Gaussian). The classic use of the Canny operator is 
a gradient method, but in some parts, it also contains elements of the Laplace approach [6, 7, 8].

The analysis of the frequency domain using Fourier transform is very useful for signal analysis because the frequency domain is very important for the consideration of the nature of the signal and the influence of the noise over it. The disadvantage of this technique is the loss of time domain information. When viewing the Fourier transform in a frequency domain, it is difficult to say when a certain event occurred, or when some frequencies occurred. To overcome this problem, only a small part ("window") of the signal is analyzed at a time, and this window slides over the signal applying the Fourier transform on it. In this way, part of the frequency information is lost in order to get an information about the time when a certain frequency has occurred. Wavelet transformation is used to solve this problem. It allows the use of different window sizes for different frequencies. The basic difference between the wavelet transformation and the short-time Fourier transform is that the window length changes at the wavelet transform and in this way the frequency and time resolution can be changed. The basic idea of each wavelet transform is to present an arbitrary function $x(t)$ as a superposition of a wavelet set or basic functions. The basic functions are derived from a prototype called mother wavelet, by scaling or translating this function. The wavelet transformation of the $x(t)$ signal is given in Equation (4) [9, 10]:

$$
\psi(\tau, s)=\frac{1}{s} \int_{-\infty}^{\infty} x(t) * \psi^{*}\left(\frac{t-\tau}{s}\right) \mathrm{d} t
$$

where $\tau$ is a translation, and $s$ is a scaling, while $\psi$ is a "mother" wavelet, and $\psi^{*}$ is conjugally complex of $\psi$. When processing images in a spatial domain, the operation is discretized. Discrete wavelet transformation (DWT) is defined as [9, 10]:

$$
D W T_{x}^{\psi}(\tau, s)=\frac{1}{\sqrt{|s|}} \int_{-\infty}^{\infty} x(t) * \psi^{*}\left(\frac{t-\tau}{s}\right) \mathrm{d} t .
$$

The discrete wavelet transformation (DWT) can be presented as a matrix $\psi(c)$ and DWT coefficients can be obtained by taking an internal product between the signal and the wavelet matrix [9, 10]:

$$
D W T_{x}^{\psi}[n, s]=\frac{1}{\sqrt{|s|}} \sum_{n} x[n] \psi_{n, s}^{*}[n] .
$$

Special family of wavelet functions has been developed for DWT. They are generally divided into orthogonal or biorthogonal and are characterized by a highpass and low-pass filters [11. Daubechies wavelet belongs to a family of orthogonal functions and the main feature is the possibility of the maximum number of vanishing moments for a predefined supported length. Types of Daubechies (db) wavelets that are most commonly used in practical applications are $\mathrm{dbN}$, where $\mathrm{N}$ represents the order as well as the number of vanishing moments in the supported interval from 0 
to 20. By increasing the order of the Daubechies wavelet, better characteristics are obtained, but the complexity of the implementation, the price of the system and errors in the calculations rise. In practical applications, orders 2 to 10 are most commonly used [10, 12, 13].

A very important advantage of the wavelet transform is that it uses a multiresolution analysis that allows analysis of different signal frequencies at different frequency resolutions. For high-frequency parts, shorter windows are used, which ensures good time domain resolution, while longer parts of the lower frequencies have longer windows, thus giving good information about the frequencies. If the signal is passed through a set of two filters, low-pass and high-pass, its frequency content will be split into two equal-width ranges. The output from these filters contains half the frequency of the original signal and the same number of samples as the original signal. By decimating, or by passing the input signal through the low-pass filter, the number of samples is halved so that the time resolution is also halved while the frequency resolution increases. The high-pass filter transmits high-frequency content, i.e. signal details. The low-pass signal transmits low-frequency content, or signal approximation. This approximation signal can be further fed through two filters and the process can be repeated until the desired decomposition level is reached. The complete information on the original signal is contained in the last approximation signal and all the detail signals [14, 15.

Higher compression ratio disrupts the quality of the image, resulting in a large loss of information, and hence makes it difficult to process them, for example, to do an edge detection or facial recognition [16, 17]. The decomposition significantly degrades the image quality, but besides this degradation there are various types of noise that further impair the image quality. To solve this problem or to control it, the various types of filters have been developed [18, [19].

In this paper we used the characteristics of wavelet transform in order to image compression, or as a method on which some algorithms for image compression is based, such as JPEG2000 and SPIHT. Images from the BSD database are compressed to the third decomposition level which resulted in a high degree of compression.

\section{SYSTEM MODEL AND PROPOSED APPROACH}

In this paper, the BSD (Berkeley Segmentation Dataset) image database was used for analysis, with its corresponding ground truth images [20]. Table 1 lists the selected images from the BSD database meeting the complexity criteria [16], that is, each image consists of a different level of detail: small, medium, and high level of detail. The number of details was calculated by making DCT and DWT on the high-frequency components (details), which are divided into four quadrants, along both directions $(x$ and $y$ ). After that, the mean absolute value of the amplitude of the components belonging to the quadrants is calculated [16]: DCT in quadrant 1 (dctd); DCT in quadrants 2 and 3 (dctm); DWT in quadrant 1 (dwtd); DWT in 
quadrants 2 and 3 (dwtm).

\begin{tabular}{|c|r|r|r|r|r|}
\hline & Images & dctd & dctm & dwtd & dwtm \\
\hline Criterion $L$ & $\# 135069$ & 1.544 & 2.517 & 0.181 & 0.354 \\
\hline Criterion $M$ & $\# 35010$ & 3.838 & 6.197 & 1.199 & 2.048 \\
\hline Criterion $H$ & $\# 8143$ & 7.868 & 15.241 & 3.181 & 6.336 \\
\hline
\end{tabular}

Table 1. Criteria

Figure 1 gives a flow diagram of the proposed approach. In the proposed approach, firstly, the BSD and the ground truth images are read on which the DWT is applied to the third decomposition level using the Daubechies wavelet (optionally from 2 to 20). Since grayscale images are needed, a conversion is made, and variables are created for filters and new images. In order to assign input values to a filter, it is necessary to perform edge detection on level 1 images. Values stored in the filter are information about location of pixels pointing at the potential edge. The compressed image on which the detection is performed is a binary image and passing through the loop, each pixel is examined. If it is equal to the 1 , that is, there is an edge, the median filter is used on the pixel and 8 neighbors of pixel. In other words, only those pixels that are relevant for the edge detection are passed through the filter. After that, the detection is applied and the results are compared, as shown in Figure 1. The algorithm is applied to all edge detection operators (Canny, LoG, Sobel, Prewitt, Robert).

The algorithm consists of the following steps:

Step 1 (Read image): Read the original image. If it is a color image, converts it to a grayscale image.

Step 2 (DWT): DWT is applied to the third decomposition level onto a read image, whereby as a result, three images are obtained: image from level 1, image from level 2 and image from level 3. The next steps in the algorithm are applied separately for an image from each level.

Step 3 (Edge detection): On level 1 image, an edge detection is used by selecting one of detectors (Canny, LoG, Sobel, Prewitt, Roberts). The image with detected edges serves to create a filter that will contain only those pixel coordinates where the edges are located.

Step 4 (Filtering image): Every pixel in the image is analyzed and if it is equal to 1 , the filter records its coordinates.

Step 5 (Filtering 8-neighbors of pixel): If the condition in step 4 is fulfilled, filtering is done by filtering 8 neighbors of pixel relative to the current pixel using the median filter (Figure 2). So, only those image pixels that can be edges are filtered. The neighbors of pixel are extracted using the following formula: 
If current pixel $P(i, j)$ is edge then use following neighbor pixels:

$$
\begin{aligned}
f\left(P_{i, j}\right)= & f\left(P_{i-1, j-1}\right), f\left(P_{i-1, j}\right), f\left(P_{i-1, j+1}\right), f\left(P_{i, j-1}\right), f\left(P_{i, j}\right), f\left(P_{i, j+1}\right), \\
& f\left(P_{i+1, j-1}\right), f\left(P_{i+1, j}\right), f\left(P_{i+1, j+1}\right)
\end{aligned}
$$

and use median filter only on those pixels.

Step 6 (Edge detection on filtered image): Edge detection is applied on a filtered image.

For filtering level 2 and level 3 images, coordinates from level 1 image are used.

The proposed algorithm has a quadratic complexity and can be represented as:

$$
O(\text { row }, c o l)=8(\text { row }-2)(\operatorname{col}-2)+1 .
$$

In Figure 3, the complexity of the algorithm is given, depending on the number of pixels in the row (row) and the number of pixel in columns (cols).

In order to accurately and precisely present how well the edge detection is done, it is necessary to calculate Precision, Specificity, Sensitivity and Accuracy or F measure [21]. F measure (F1 score) is a harmonic mean of precision and recall and it combines precision and recall according to the formula [21]:

$$
F=\frac{2 * \text { Precision } * \text { Recall }}{\text { Precision }+ \text { Recall }} \times 100
$$

$F$ is within the limits of $0 \leq F \leq 1$, ideally, $F$ is equal to 1 . In the results, $F$ is multiplied by 100 and represents a percentage value.

Figure of Merit (FoM) was proposed by Pratt [22] and is a measure for estimating the accuracy of detected edges. In other words, it represents the deviation of the actual (calculated) point of the edge from the ideal point of the edge, and is defined as:

$$
F_{O} M=\frac{1}{\max \left[I_{d}, I_{i}\right]} \sum_{k=1}^{I_{d}} \frac{1}{1+\delta e^{2}(k)}
$$

where $I_{d}$ is the number of points on the detected edge, and $I_{i}$ is the number of points on the ideal edge, $e(k)$ represents the distance between the detected edge and the ideal edge, and $\delta$ is scaling constant and is usually $1 / 9$. FoM is within $0 \leq F_{O} M \leq 1$. In this case, $F_{O} M$ is multiplied by 100 and represents a percentage value. The higher the $F_{O} M$ is, the better the detected edge is [22].

As an objective measure of the edge detection credibility, Performance Ratio (PR) was used too. The PR is ideally equal to infinity. The PR is calculated as the ratio of the true edges to false ones [23, 24]: 
$P R=\frac{\text { True Edge(Edge pixels identified as Edges })}{\text { False Edges(Non Edge pixels identified as Edges })+(\text { Edge pixels identified as Non Edge pixels })} \times 100$.

\section{RESULTS}

\subsection{F Measure}

Tables 2, 3 and 4 show the $\mathrm{F}$ values before and after the application of the proposed approach on image with a small, medium and high number of details, respectively, over which $\mathrm{db}$ (from $2^{\text {nd }}$ to $10^{\text {th }}$ order) wavelet transform to the third decomposition level was applied. These tables show the values obtained for the five operators.

From Table 2 it can be seen that based on the obtained results, in the first decomposition level, using the proposed approach, improvements were achieved with the Canny operator in almost all cases, except for db4. A similar situation occurs when a LoG operator is used, with the exception that improvements have not been achieved in the case of db6. Using the proposed approach and image with low details, significant improvements have been achieved at the second level, where all operators record improvements in $F$ values, except in the case of db8 with Prewitt and Sobel operators. In the third level, only Canny and LoG record improvements in $\mathrm{F}$ values using the proposed approach.

In the image with a medium number of details, very small improvements have been achieved in some operators at the first level, or the values are generally similar, without major deviations, as can be seen in Table 3. The situation is different with the second and third decomposition levels, where improvements are achieved by all operators, with the difference that improvements are higher in the third level.

In the case where the image consists of a high number of details, using the proposed approach, a similar or slightly better $\mathrm{F}$ value is obtained. At the second level, improvements are generally achieved with gradient operators, while at the third level, using the proposed approach, better values are obtained for all operators. 


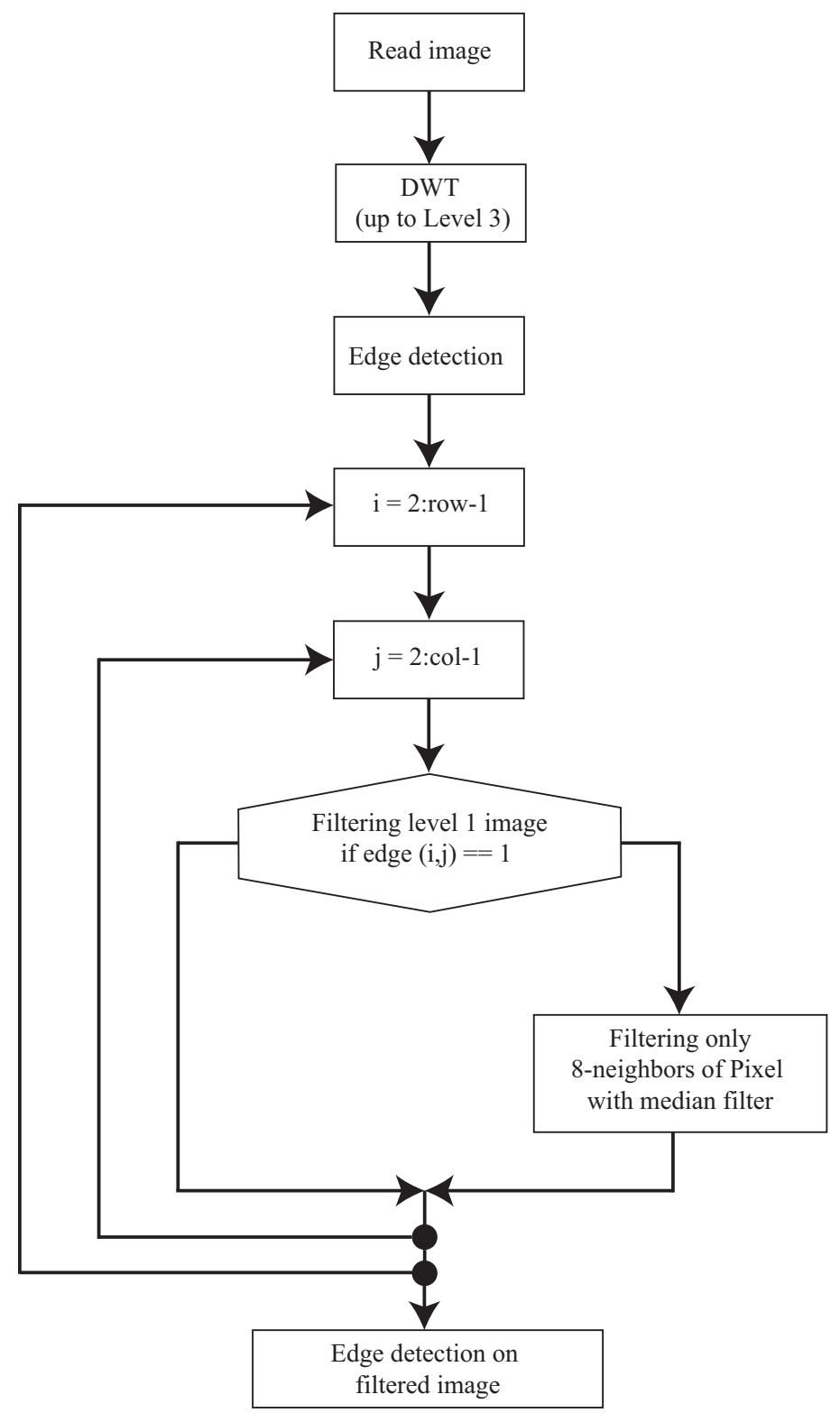

Figure 1. Proposed approach 


\begin{tabular}{|l|l|l|}
\hline$P_{i-1, j-1}$ & $P_{i-1, j}$ & $P_{i-1, j+1}$ \\
\hline$P_{i, j-1}$ & $P_{i, j}$ & $P_{i, j+1}$ \\
\hline$P_{i+1, j-1}$ & $P_{i+1, j}$ & $P_{i+1, j+1}$ \\
\hline
\end{tabular}

Figure 2. Extraction neighbors of pixel

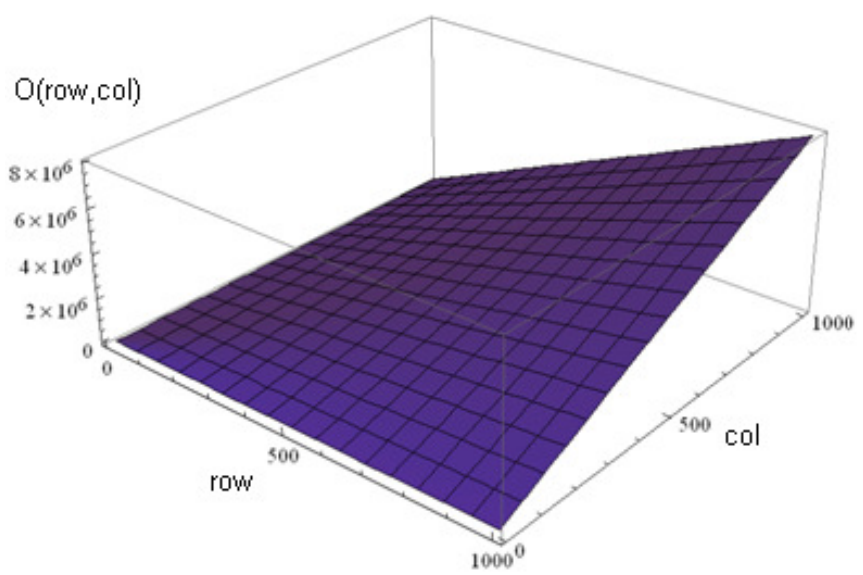

Figure 3. Complexity of proposed algorithm 


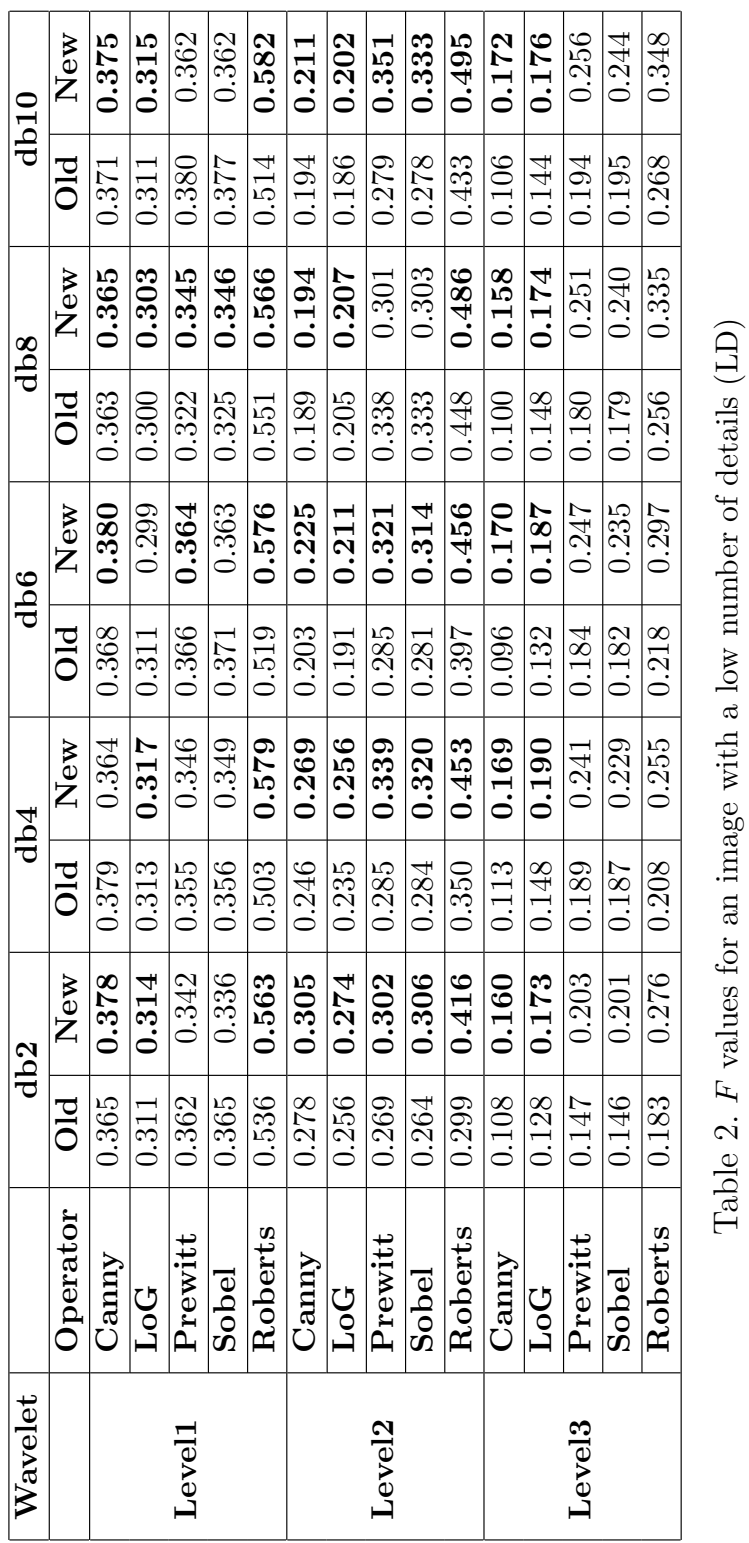




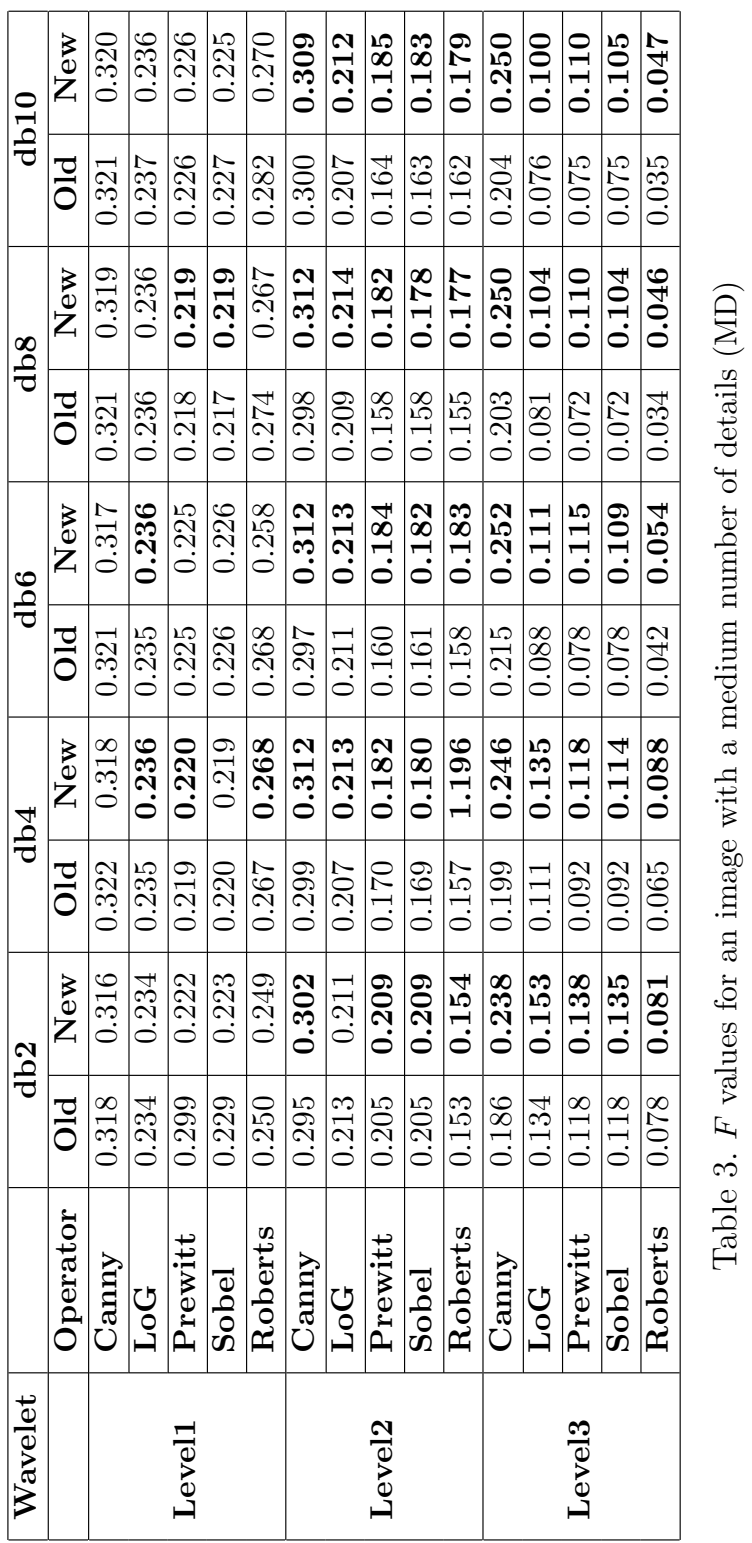




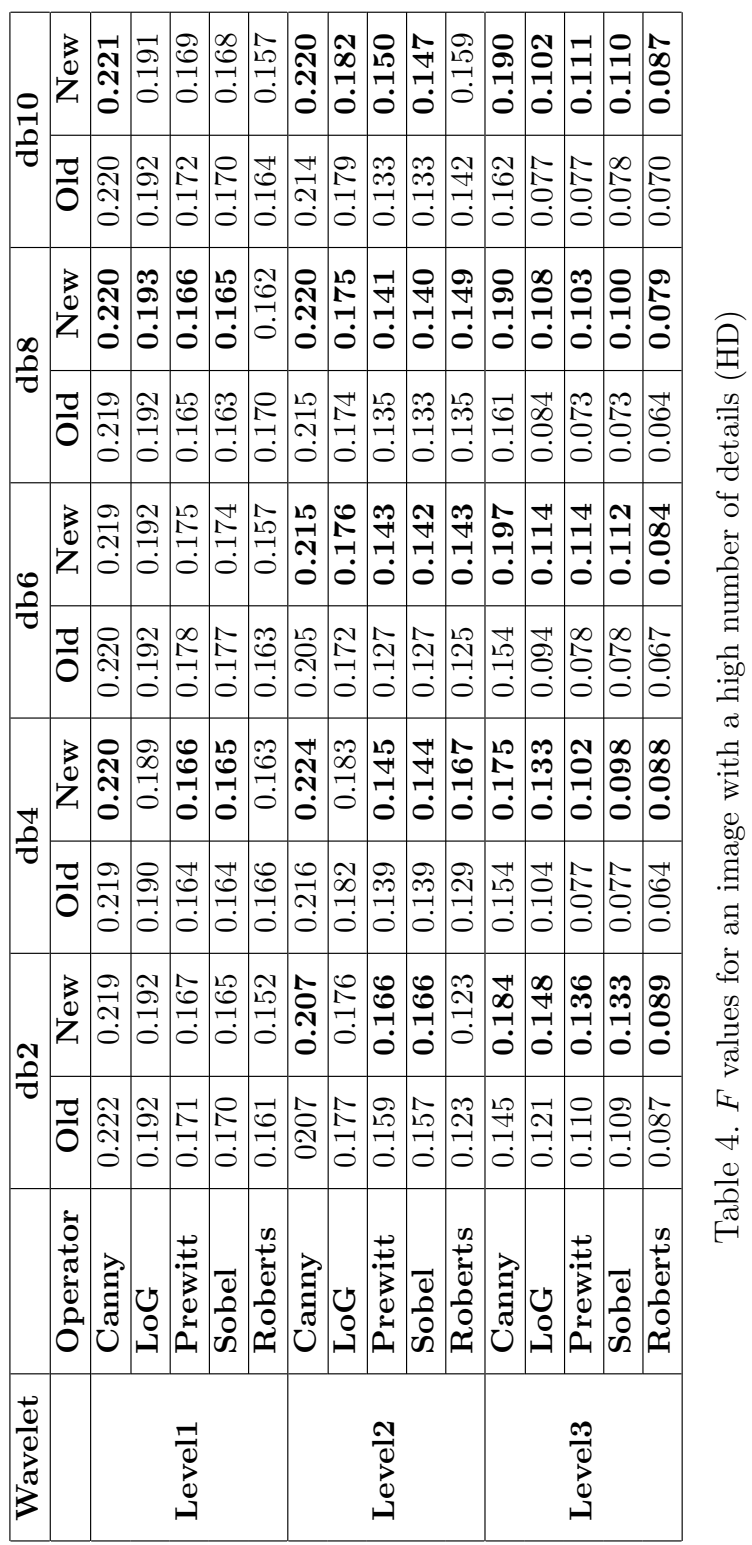




\subsection{Figure of Merit}

Tables 5, 6 and 7 show the FoM values before and after the application of the proposed approach on image with a small, medium and high number of details, respectively, over which $\mathrm{db}$ (from $2^{\text {nd }}$ to $10^{\text {th }}$ order) wavelet transform to the third decomposition level was applied. These tables show the values obtained for the five operators.

From Table 5 it can be seen that in the first decomposition level, the best values are obtained for the Canny operator, where in almost all cases it gives better values, except in the case of db4. By comparing Table 5 with Table 2, it can be seen that using FoM objective measurements, improvements have been made in the same or similar cases. By increasing the compression, that is, in Levels 2 and Levels 3, using the proposed approach, better FoM values were obtained, and greater improvements were achieved, especially in the third level.

In the case of an image with a medium number of details, in the first level, Canny gave the best results, in other words, better values were achieved. In the second level, in the case of $\mathrm{db} 2$, improvements were achieved only with the Canny operator, while other operators gave similar, but lower values. In other cases, the proposed algorithm records substantially better FoM values. For images with a medium number of details, the best edge detection enhancements have been achieved in the third level using the proposed algorithm.

Since the compression ratio is higher in the image with a high number of details, based on this fact, it can be concluded that the detection will be much worse. Also, in the case of images with a high number of details, the best improvements are achieved with the Canny operator at all levels. Based on the results obtained, it can be seen that the best improvements are achieved in the third level.

\subsection{Performance Ratio}

Tables 8,9 and 10 show PR values before and after applying the proposed approach using five edge detection operators and three wavelet decomposition levels. Table 8 shows the values for an image with a low number of details. Based on the results obtained, it appears that improvements have been made with certain operators. However, based on the values obtained in Table 8, it can be seen that the old $\mathrm{PR}$ values and new PR values obtained using the proposed approach have greatest difference when using the Robert operator.

Table 9 contains the PR values obtained for an image with medium number of details. From Table 9 can be seen that the new values obtained are best in the second and third decomposition levels. In other words, the best improvements are achieved in the second and third levels, depending on the operator used and the order of the $\mathrm{db}$ wavelet.

Table 10 contains PR values obtained for an image with a high number of details. Based on these results, it can be seen that improvements have been achieved using the proposed approach, especially in the third decomposition level. 


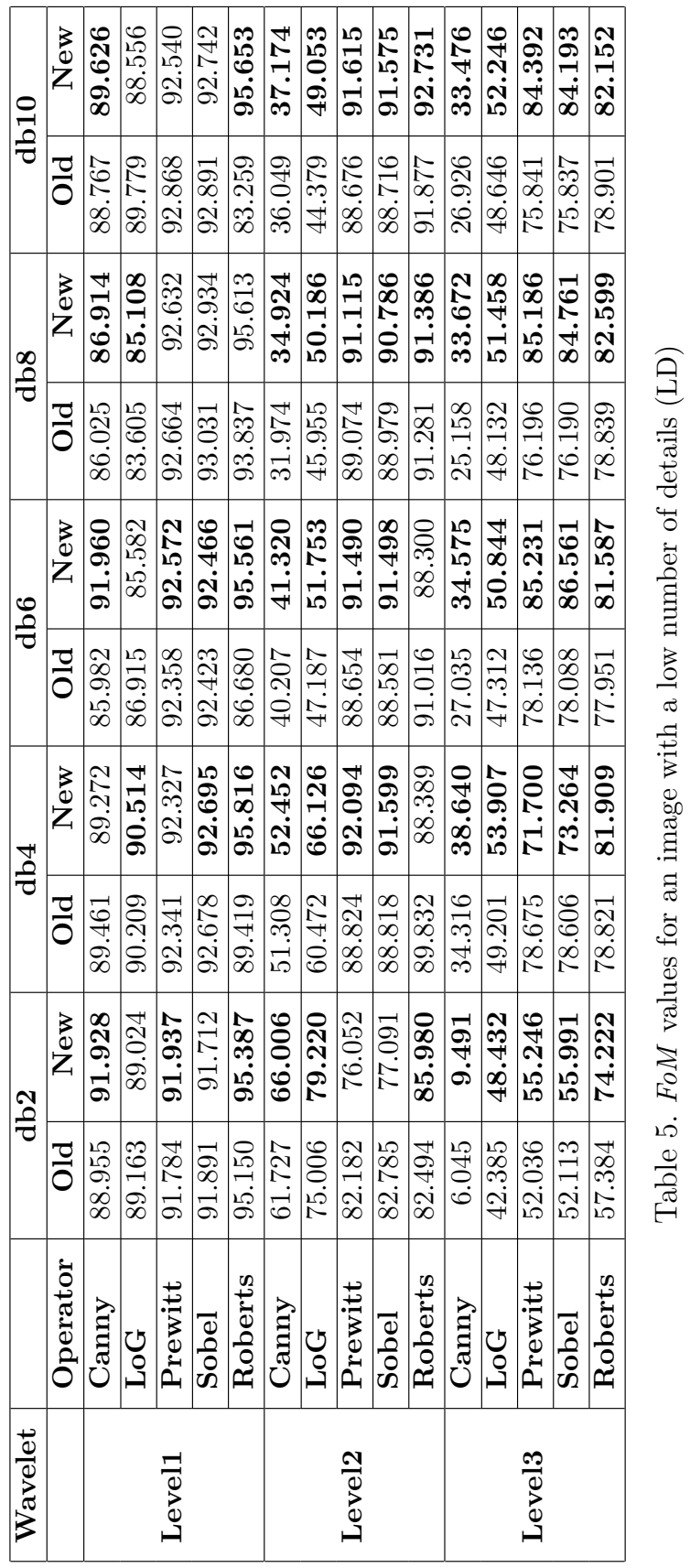




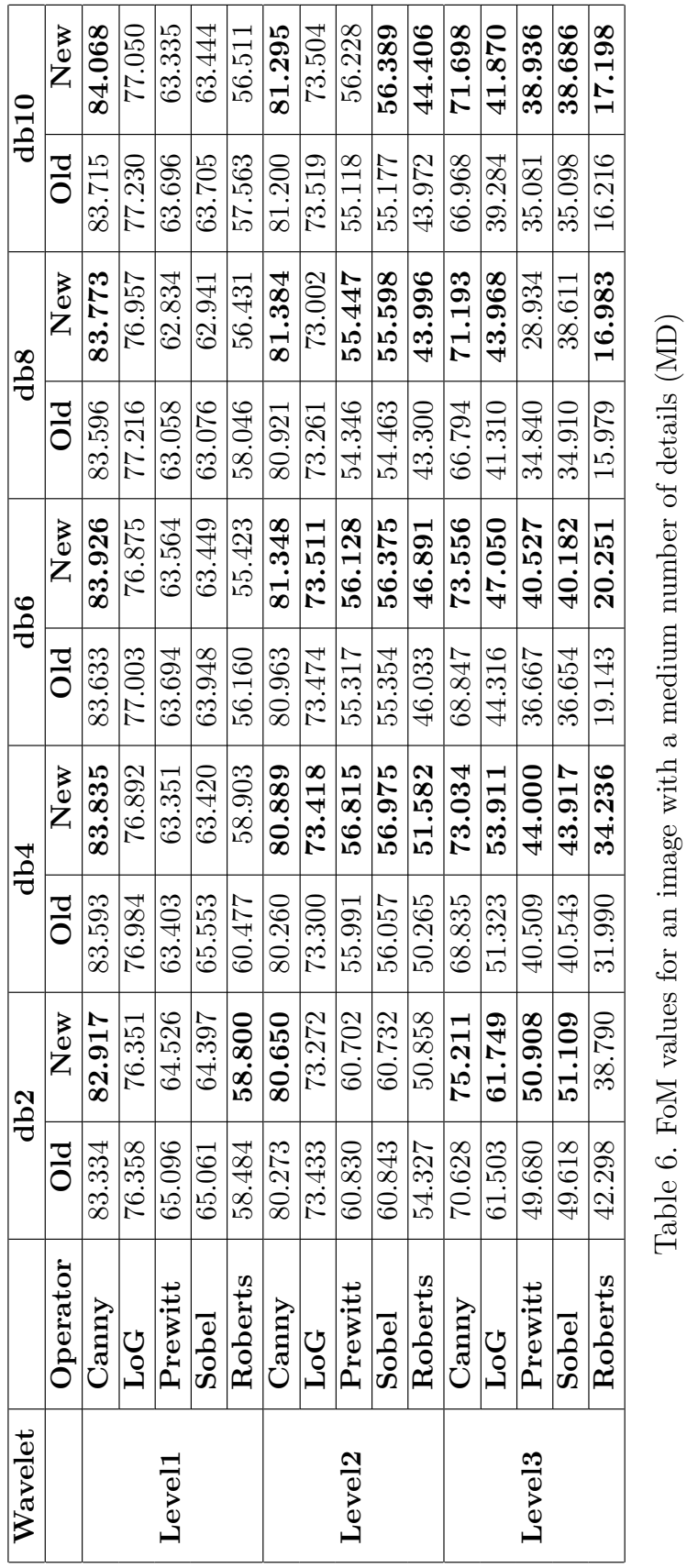




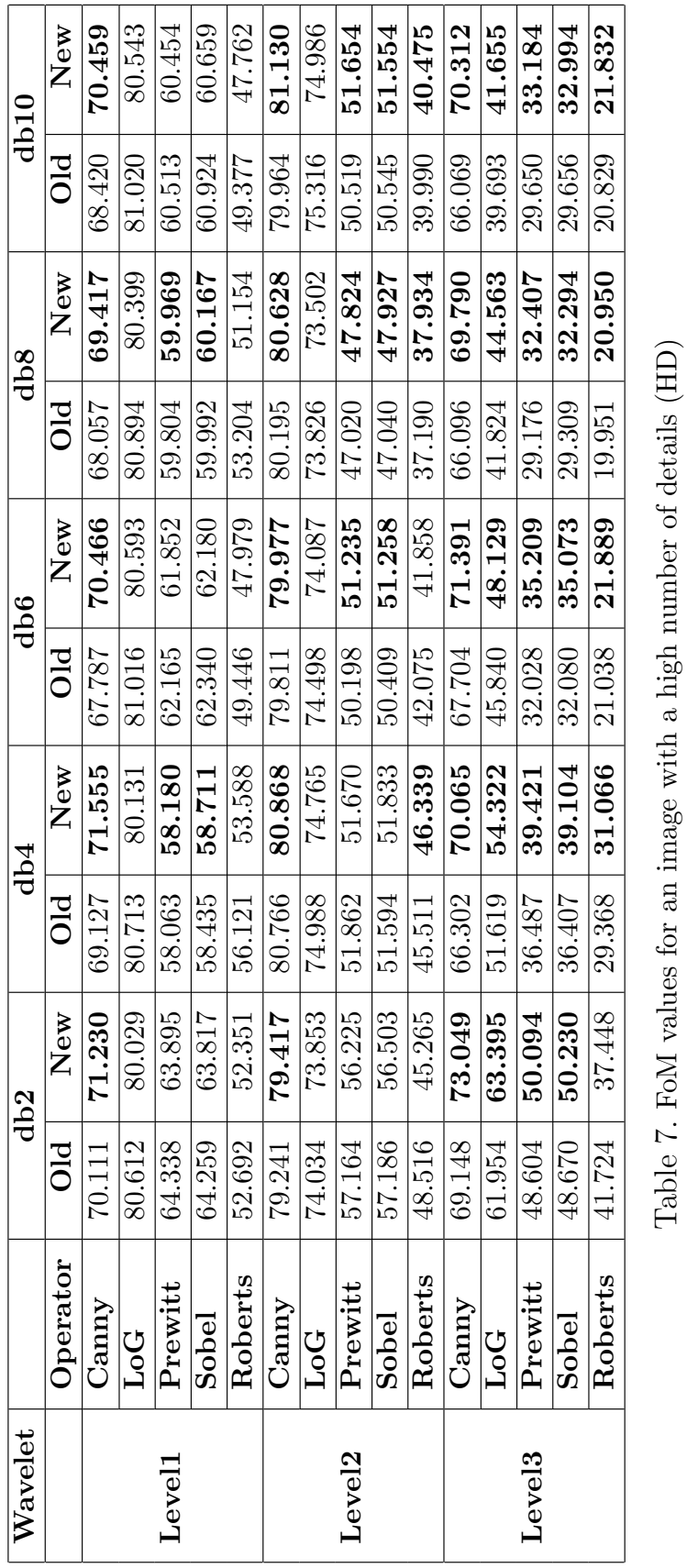




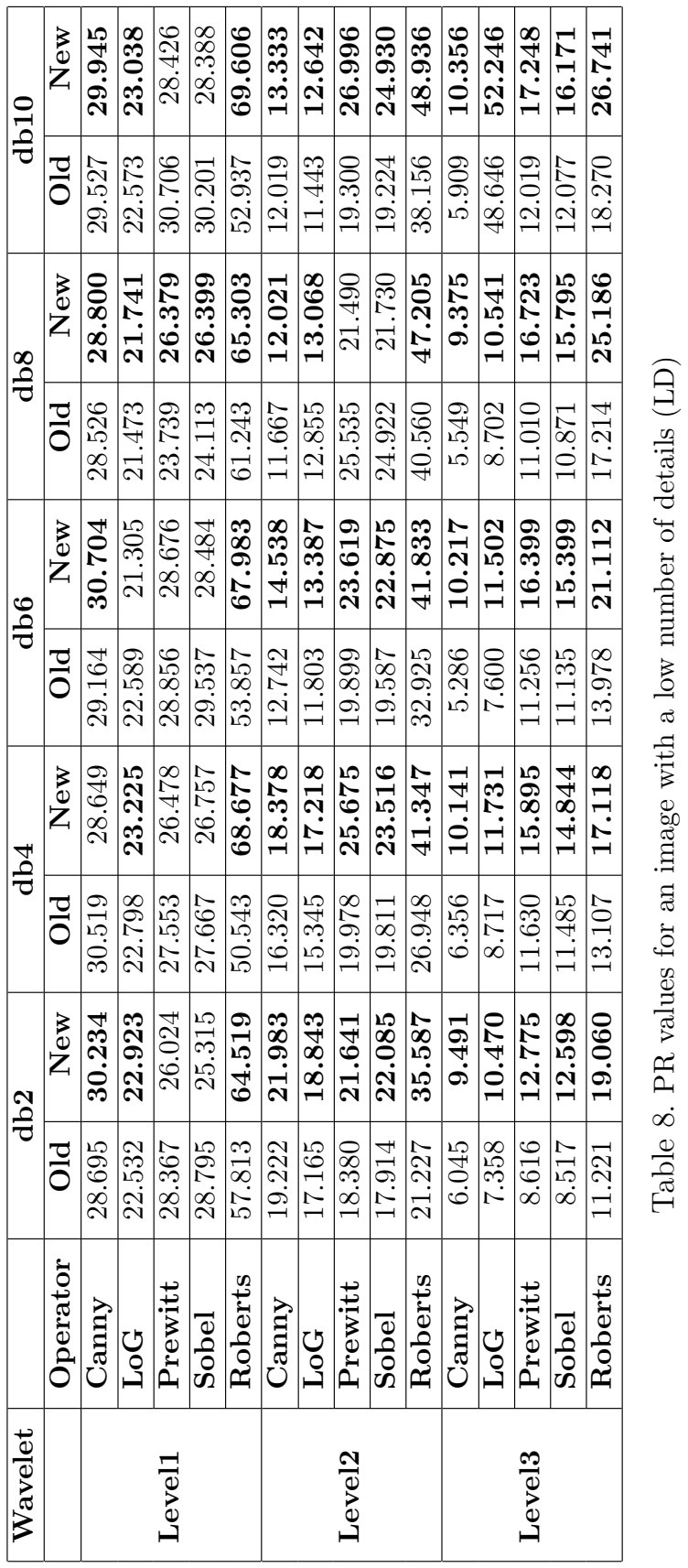




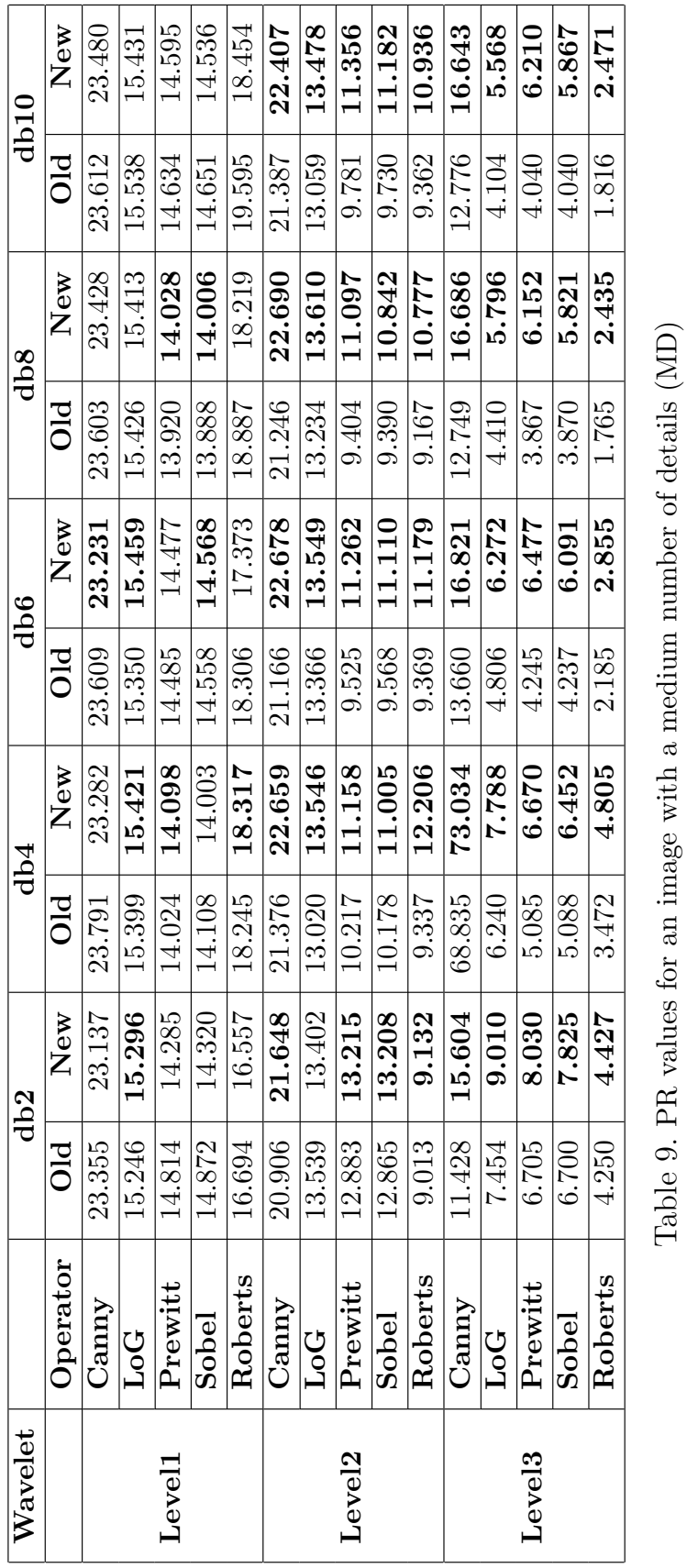




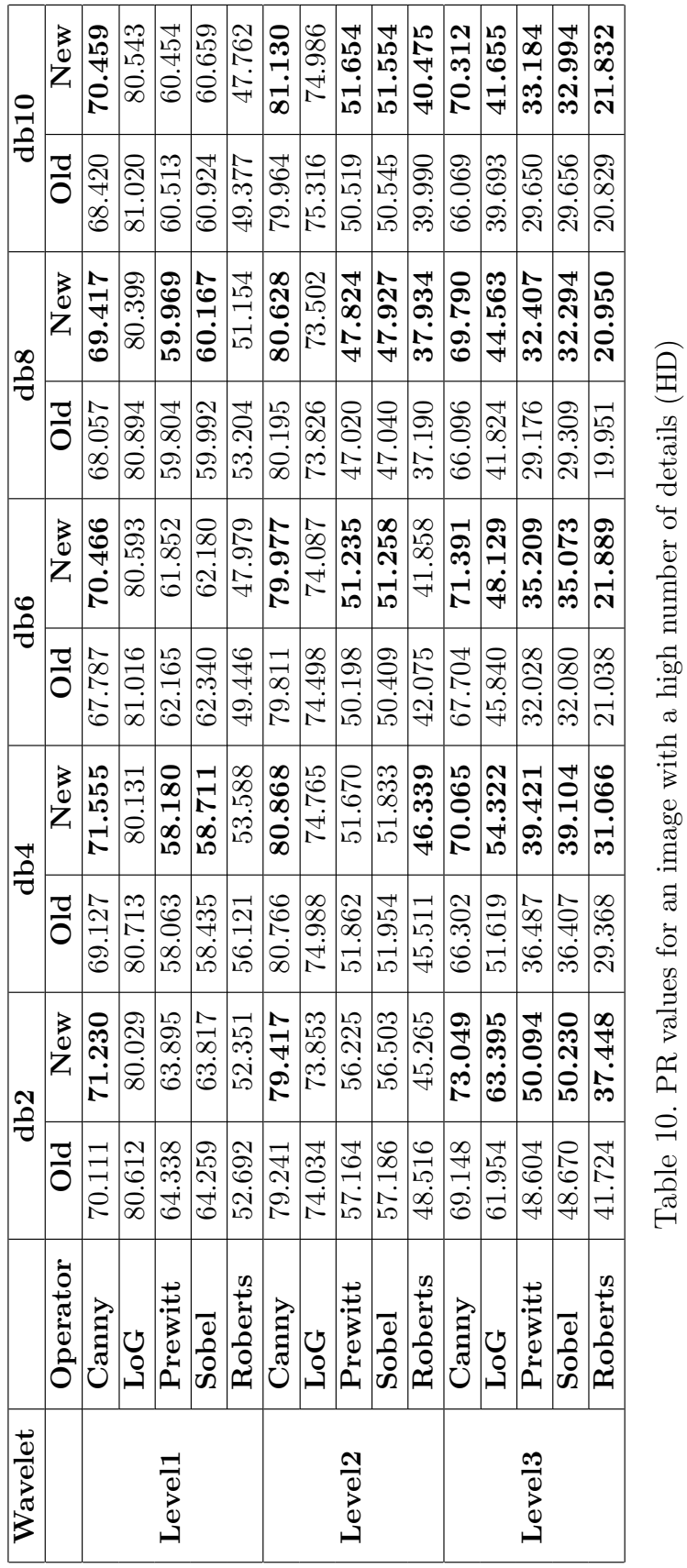




\section{CONCLUSIONS}

This paper proposed a new approach to edge detection in the images on which the wavelet decomposition was applied to the third level. As mother wavelet, Daubechies was used from the second to the tenth order. The analyzed images are categorized into three complexity criteria, so they consist of a small, medium and high number of details. F measure, FoM, and PR were used for an objective measure. The proposed approach provides significant improvements in edge detection for almost all operators (Canny, LoG, Prewitt, Sobel).

Depending on the number of details in the image, the decomposition level as well as db wavelet, the improvements are different. With a small number of details, the greatest improvements were achieved with the Canny operator. Other operators also achieved improvement but depending on the db wavelet order. Based on the obtained results, in the image with a small number of details, it can be seen that best improvements are achieved in the third level using Laplacian operators. In the image with the medium number of details, in the first level similar results are generally obtained, while in the second and the third levels improvement is made using the proposed approach. For images with a high number of details, better or similar values are obtained using the proposed approach. What can be concluded is that in the second level, the best values are obtained by gradient operators.

Considering that the compression ratio is higher of the image with a higher number of details, based on this fact, it can be concluded that the detection will also be poorer, and consequently there will be a lower $\mathrm{F}$ values, FoM values and $\mathrm{PR}$ values. Since the proposed approach is intended for systems where compression is used, i.e. compressed image processing, it can be concluded that increasing the degree of compression also provides a better difference, or better value using the proposed approach.

Today's systems require image quality to be as good as possible, with as much compression as possible in order to process these images in real time, such as edge detection, segmentation, streaming, streaming in systems using augmented reality, etc. The proposed approach can find many practical applications in all systems where real-time information needs to be processed, especially in television systems, but it also provides a good basis for the direction of future research related to compression and edge detection.

\section{Acknowledgment}

This work was done within the research project of the Ministry of Science and Technological Development of Serbia TR35026 and III47016. 


\section{REFERENCES}

[1] Chen, Y.-Wang, D.-Bi, G.: An Image Edge Recognition Approach Based on Multi-Operator Dynamic Weight Detection in Virtual Reality Scenario. Cluster Computing, Vol. 22, 2018, Suppl. 4, pp. 8069-8077, doi: 10.1007/s10586-017-1604-y.

[2] Srivastava, D.-Kohli, R.-Gupta, S.: Implementation and Statistical Comparison of Different Edge Detection Techniques. In: Bhatia, S., Mishra, K., Tiwari, S., Singh, V. (Eds.): Advances in Computer and Computational Sciences. Springer, Singapore, Advances in Intelligent Systems and Computing, Vol. 553, 2017, pp. 211-228, doi: 10.1007/978-981-10-3770-2_20.

[3] Maini, R.-Aggarwal, H.: Study and Comparison of Various Image Edge Detection Techniques. International Journal of Image Processing, Vol. 3, 2009, No. 1, pp. $1-11$.

[4] Chaganti, V.R.: Edge Detection of Noisy Images Using 2-D, Discrete Wavelet Transform. Thesis, Florida State University Libraries, 2005.

[5] Dong, X.-Li, M.-Mino, J.-Wang, Z.: Edge Detection Operator for Underwater Target Image. 2018 IEEE $3^{\text {rd }}$ International Conference on Image, Vision and Computing (ICIVC), 2018, pp. 91-95, doi: 10.1109/ICIVC.2018.8492749.

[6] Kunng, T.-Zhu, Q-X.-Sun, Y.: Edge Detection for Highly Distorted Images Suffering Gaussian Noise Based on Improve Canny Algorithm. Kybernetes, Vol. 40, 2011, No. 5-6, pp. 883-893, doi: 10.1108/03684921111142430.

[7] Jun, L.: A Wavelet Approach to Edge Detection. Thesis, Sam Houston State University, Huntsville, Texas, 2003.

[8] Muthukrishnan, R.-Radha, M.: Edge Detection Techniques for Image Segmentation. International Journal of Computer Science and Information Technology, Vol. 3, 2011, No. 6, pp. 259-267, doi: 10.5121/ijcsit.2011.3620.

[9] Yang, T.-Sun, G.-Dunn, X.: A New Method of Wavelet Transform-Based Edge Detection. 2011 IEEE $13^{\text {th }}$ International Conference on Communication Technology, Jinan, 2011, pp. 789-792, doi: 10.1109/ICCT.2011.6157985.

[10] Yelampalli, P.K.R.-Nayak, J.-Gaidhane, V.H.: Daubechies WaveletBased Local Feature Descriptor for Multimodal Medical Image Registration. IET Image Processing, Vol. 12, 2018, No. 10, pp. 1692-1702, doi: 10.1049/ietipr.2017.1305

[11] Wang, S.-H.-Zhang, Y.-D.-Dong, Z.-Phillips, P.: Wavelet Families and Variants. Chapter 6. In: Wang, S.-H. et al.: Pathological Brain Detection. Springer, Singapore, Brain Informatics and Health, 2018, pp. 85-104, doi: 10.1007/978-981-104026-9_6.

[12] Balachandran, A.-Ganesan, M.-Sumesh, E. P.: Daubechies Algorithm for Highly Accurate ECG Feature Extraction. 2014 International Conference on Green Computing Communication and Electrical Engineering (ICGCCEE), Coimbatore, pp. 1-5, 2014, doi: 10.1109/ICGCCEE.2014.6922266.

[13] Rana, K.-Thakur, S.: Comparisons of Wavelets and Algorithms Based on Wavelets and Comparing the Results with JPEG. 2017 International Conference on 
Energy, Communication, Data Analytics and Soft Computing (ICECDS), Chennai, 2017, pp. 3871-3876, doi: 10.1109/ICECDS.2017.8390189.

[14] Zhao, D.-Sun, G.: A Novel Edge Detection Approach Using Multi-Resolution Image. $2^{\text {nd }}$ International Conference on Computer Engineering and Technology, Chengdu, 2010, pp. V2-692-V2-695, doi: 10.1109/ICCET.2010.5485706.

[15] RaO, G. S. B.-Prasad, P. M. K.-Kumar, M. N. V. S. S.: Investigation of Various Orthogonal Wavelets for Precise Analysis of X-Ray Images. Journal of Engineering Research and Applications, Vol. 5, 2015, No. 2, Part 3, pp. 24-32.

[16] Ilic, S.-Petrovic, M.-Jaksic, B.-Spalevic, P.-Lazic, L.-MiloseVIC, M.: Experimental Analysis of Picture Quality after Compression by Different Methods. Przegląd Elektrotechniczny, Vol. 89, 2013, No. 11, pp. 190-194.

[17] Delac, K.-Grgic, M.-Grgic, S.: Effects of JPEG and JPEG2000 Compression on Face Recognition. In: Singh, S., Singh, M., Apte, C., Perner, P. (Eds.): Pattern Recognition and Image Analysis (ICAPR 2005). Springer, Berlin, Heidelberg, Lecture Notes in Computer Science, Vol. 3687, 2005, pp. 136-145, doi: 10.1007/11552499_16.

[18] Rani, K. S.-RAO, D. N.: A Comparative Study of Various Noise Removal Techniques Using Filters. Research and Reviews: Journal of Engineering and Technology, Vol. 7, 2018, No. 2, pp. 47-52.

[19] Wen, X.-Deng, Z.-Xue, H.: An Improved Median Filtering Image De-Noising Algorithm. Revista Tecnica de la Facultad de Ingenieria Universidad del Zulia, Vol. 39, 2016, No. 5, pp. 293-298, doi: 10.21311/001.39.5.38.

[20] The Berkeley Segmentation Dataset and Benchmark. Available at: https://www2. eecs.berkeley.edu/Research/Projects/CS/vision/bsds/, accessed: 14.02.2019.

[21] Arbeláez, P.-Maire, M.-Fowlkes, C.-Malik, J.: Contour Detection and Hierarchical Image Segmentation. IEEE Transactions on Pattern Analysis and Machine Intelligence, Vol. 33, 2011, No. 5, pp. 898-916, doi: 10.1109/TPAMI.2010.161.

[22] Hagara, M.-Kubinec, P.: About Edge Detection in Digital Images. Radioengineering, Vol. 27, 2018, No. 4, pp. 919-929, doi: 10.13164/re.2018.0919.

[23] Khaire, P. A.-Thakur, N. V.: A Fuzzy Set Approach for Edge Detection. International Journal of Image Processing (IJIP), Vol. 6, 2012, No. 6, pp. 403-412.

[24] Jabbar, S. I.-Day, C. R.-Heinz, N.-Chadwick, E. K.: Using Convolutional Neural Network for Edge Detection in Musculoskeletal Ultrasound Images. Proceeding of the 2016 International Joint Conference on Neural Networks (IJCNN), pp. 4619-4626, 2016, doi: 10.1109/IJCNN.2016.7727805. 


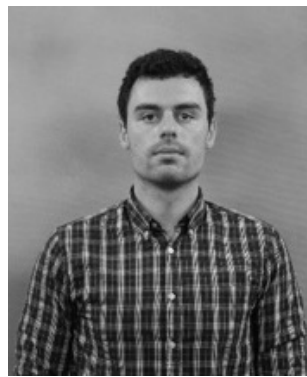

Vladimir MAKsIMović received his B.Sc. and M.Sc. degrees in electrical engineering from the Faculty of Technical Sciences in Kosovska Mitrovica, University of Priština, Serbia. He is Ph.D. candidate at the same faculty and his areas of research include image processing, telecommunications and multimedia systems. He has authored several scientific papers.

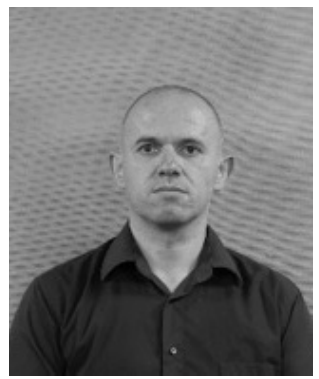

Branimir J AKŠI ́́ received his B.Sc. and M.Sc. degrees in electrical engineering from the Faculty of Technical Sciences in Kosovska Mitrovica, University of Priština, Serbia, and his Ph.D. degree in electrical engineering from the Faculty of Electronic Engineering, University of Niš, Serbia in 2015. He is Assistant Professor at the Faculty of Technical Sciences in Kosovska Mitrovica. Areas of his research include telecommunications and multimedia systems. He has authored over 70 scientific papers on the above subject.

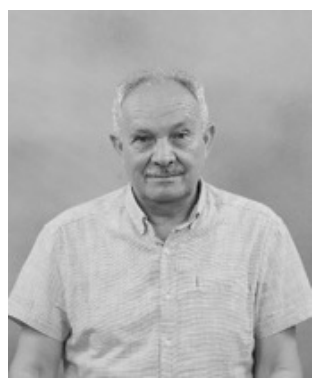

Mile Petrović is Full Professor at the Department of Electronics and Telecommunications Engineering at the Faculty of Technical Sciences in Kosovska Mitrovica, Serbia. He has extensive experience in digital broadcasting systems and multimedia applications, mobile technology and digital image processing. He is the author of over 100 scientific peer-reviewed papers, initiator of a number of international Tempus projects in the country and certified patents. He has participated in scientific research projects as well as in projects for the modernization and enhancing quality of higher education. He is author of many textbooks and scientific articles in the field of mobile radio and telecommunication networks.

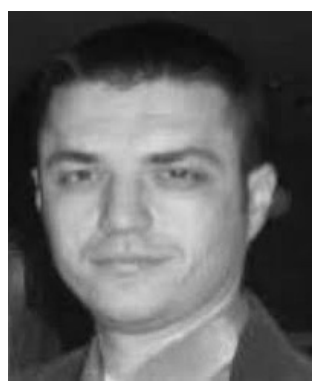

Petar SPALEvić received his B.Sc. degree from the Faculty of Electronic Engineering, University of Priština, in 1997 and his M.Sc. and Ph.D. degrees from the Faculty of Electronic Engineering, University of Niš in 1999 and 2003, respectively. He is Professor at the Faculty of Technical Sciences, Department of Telecommunications in Kosovska Mitrovica. His primary research interests are statistical communications theory, optical and wireless communications, applied probability theory and optimal receiver design. 


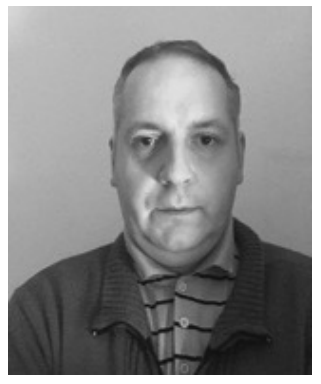

Stefan PaNIĆ received his M.Sc. and Ph.D. degrees in electrical engineering from the Faculty of Electronic Engineering, Niš, Serbia, in 2007 and 2010, respectively. His research interests in mobile and multi-channel communications include statistical characterization and modelling of fading channels, performance analysis of diversity combining techniques, outage analysis of multi-user wireless systems subject to interference. Within digital communication, his current research interests include the information theory, source and channel coding, and signal processing. He has published over 40 SCI indexed papers. Currently he is Associated Professor at the Department of Informatics, Faculty of Natural Science and Mathematics, University of Priština. 